\title{
DUMMETT ON INDEFINITE EXTENSIBILITY
}

\author{
Øystein Linnebo \\ University of Oslo
}

\begin{abstract}
Dummetts notion of indefinite extensibility is influential but obscure. The notion figures centrally in an alternative Dummettian argument for intuitionistic logic and anti-realism, distinct from his more famous, meaning-theoretic arguments to the same effect. Drawing on ideas from Dummett, a precise analysis of indefinite extensibility is proposed. This analysis is used to reconstruct the poorly understood alternative argument. The plausibility of the resulting argument is assessed.
\end{abstract}

\section{Introduction}

Dummett spent much of his career arguing that the correct logic is intuitionistic, not classical. He also defended a form of anti-realism which he connected closely with the correctness of intuitionistic logic. His argument for these twin theses is based on a cluster of challenges to the dominant truth-conditional approach to meaning pioneered by Frege and a defense of an alternative non-Fregean approach whose central notion is proof rather than truth. As developed in the classic (Dummett, 1978), the argument is based on the famous manifestation and acquisition challenges, which have received a great deal of attention in the subsequent philosophical literature.

Beginning in (Dummett, 1963) and culminating in (Dummett, 1991), Dummett develops an alternative argument for some similar theses. As Richard Heck observes, this alternative argument is

quite different in character from the meaning-theoretic arguments for which Dummett is well known. It is a "local" argument for anti-realism about mathematics, one which depends upon considerations peculiarly mathematical in character; it therefore has not the propensity to generalize which the meaning-theoretic arguments have. ${ }^{1}$

\footnotetext{
${ }^{1}$ See (Heck, 1993, p. 233). When Peter Sullivan later repeats this interpretation, Dummett writes that
} 
While the more familiar arguments are based on global considerations about language and meaning, the alternative argument is based on the notion of indefinite extensibility, which has its home in the philosophies of logic and mathematics - although, as we shall see, it is also of broader metaphysical significance. The alternative argument is therefore local rather than global. If successful, it would establish the correctness of intuitionistic logic for parts of mathematics and possibly also other domains that are intertwined with mathematics.

My plan is as follows. I begin by explaining and analyzing the notion of indefinite extensibility $(\S \S 2-6)$. Then, I use this analysis to reconstruct Dummett's alternative argument $(\S \S 7-8)$. The reconstruction is based on a charitable interpretation of relevant writings by Dummett. I shall not insist that Dummett had the resulting argument explicitly in mind; indeed, I suspect he did not. But I believe my reconstruction is a reasonable and interesting way to develop his various remarks. ${ }^{2}$ Finally, I attempt to assess the resulting argument ( $§ 9$ ). While the argument merits serious consideration, I cannot endorse it, at least not as it stands. But I argue that the argument is more promising as an attack on classical logic than as a defense of any interesting form of anti-realism.

\section{The emergence of the notion of indefinite extensibility}

It is useful to approach the notion of indefinite extensibility by considering the intellectual context in which it emerged.

Around 1900, some paradoxes shook the foundations of mathematics. The most famous example is Russell's paradox of 1901, which asks us to consider all the sets. Some of these sets are elements of themselves (e.g., the set of infinite sets), while others are not (e.g., the set of natural numbers). So it seems possible to consider the set $R$ of sets that are not elements of themselves. From this characterization of $R$, however, we easily derive the contradiction that $R$ is an element of itself if and only if $R$ is not an element of itself. What to do?

According to a famous diagnosis by Russell himself, paradoxes such as the one just mentioned

Sullivan "is of course right to support Richard Heck's judgement that it is a different argument from that I had given previously" (Dummett, 2007, p. 786).

${ }^{2}$ To paraphrase Kripke's famous discussion of Wittgenstein on rule following and private language, I shall present Dummett's argument "as it struck me, as it presented a problem for me" (Kripke, 1982, p. 5). 
result from the fact that $[\ldots]$ there are what we may call self-reproductive processes and classes. That is, there are some properties such that, given any class of terms all having such a property, we can always define a new term also having the property in question. Hence we can never collect all of the terms having the said property into a whole; because, whenever we hope we have them all, the collection which we have immediately proceeds to generate a new term also having the said property. (Russell, 1906, p. 36; underlining added)

Clearly, Russell characterization of the notion of a "self-reproductive class" falls short of being a proper definition. It avails itself of a number of unexplained notions, which I have underlined: 'class', 'whole', and 'collection'; elsewhere, 'totality' too is thrown into the mix. The need for greater clarity is thus severe. But it is hard to deny that Russell succeeds at least in gesturing at an important idea. There are certain properties that are "self-reproductive" in the sense that, whenever some instances have been circumscribed, this very circumscription can be used to define a new instance of the property, beyond the ones that we have circumscribed. It follows immediately that it is impossible to circumscribe all the instances of the property; in this sense, the property lacks a "totality".

One good example of a property Russell regards as "self-reproductive" is that of a set. Assume we have circumscribed some sets. Then we can use the sets thus circumscribed to define yet another set, namely the set of all and only those of our circumscribed sets that are not elements of themselves. Call the resulting set $R$. On pain of contradiction, $R$ cannot belong to the sets that we circumscribed. Another instructive example is the property of being an ordinal. Assume we have circumscribed some ordinals. Put them in their natural ordering and fill in any gaps. In this way, we circumscribe an initial segment of the ordinals. Since this initial segment is a well-ordering, it has a well-order type, i.e. an ordinal. But as the Burali-Forti paradox shows, this ordinal cannot be among the ordinals that we circumscribed but must be larger than each of these.

According to Russell (1906, 1908), all the set theoretic and semantic paradoxes involve "self-reproductive" concepts, which thus include philosophically imporant concepts such as proposition, property, and definition.

Citing Russell's analysis, Dummett $(1963,1981,1991)$ characterizes a concept as indefi- 
nitely extensible just in case, for any definite collection $X$ of instances of the concept, there is another instance which is not a member of $X$.

A concept is indefinitely extensible if, for any definite characterization of it, there is a natural extension of this characterization, which yields a more inclusive concept; this extension will be made according to some general principle for generating such extensions, and, typically, the extended characterization will be formulated by reference to the previous, unextended, characterization (Dummett, 1963, pp. 195-196; underlining added)

Elsewhere, Dummett writes that a concept is indefinitely extensible just in case, whenever we can form a definite conception of a totality all of whose members fall under that concept, we can, by reference to that totality characterize a larger totality all of whose members fall under it (Dummett, 1993, p. 441; underlining added)

Notice that Dummett, just like Russell, avails himself of several unexplained notions which I have underlined, namely 'definite characterization', as well as 'totality' and a 'definite conception' thereof. We shall shortly attempt to be more precise.

\section{$3 \quad$ Panacea or hopeless obscurity?}

Reactions to the notion of indefinite extensibility have been sharply divided.

Some regard the notion (or its Russellian predecessor) as a panacea for a wide range of logical and metaphysical ills. Here are some of the claims made on behalf of the notion.

(a) It is the key to solving the logical paradoxes. ${ }^{3}$

(b) It is the key to an account of legitimate set formation and permissible abstraction more generally. ${ }^{4}$

(c) It gives rise to an argument against the possibility of generalizing over absolutely everything. ${ }^{5}$

\footnotetext{
${ }^{3}$ See e.g. (Russell, 1906), (Russell, 1908), and (Priest, 1994).

${ }^{4}$ See e.g. (Cantor, 1883), Cantor's 1897 and 1899 letters to Hilbert and Dedekind (available in (Ewald, 1996, pp. 927, 931-932)), (Russell, 1908), (Dummett, 1991), and (Shapiro, 2003).

${ }^{5}$ See e.g. (Parsons, 1974), (Glanzberg, 2004), (Fine, 2006), and (Studd, 201).
} 
(d) It gives rise to an argument for intuitionistic logic and anti-realism. ${ }^{6}$

Our present concerns are (b) and (d). ${ }^{7}$

Others complain that the notion of indefinite extensibility is hopelessly obscure and devoid of theoretical value. While (Dummett, 1991, p. 317) laments that "Frege did not have a glimmering of a suspicion of the existence of indefinitely extensible concepts", George Boolos, in an influential critical discussion, retorts:

To his credit, Frege did not have a glimmering of a suspicion of the existence of indefinitely extensible concepts. (Boolos, 1998, p. 224)

Others have followed suit:

I, like Boolos, have no use for Michael Dummett's notion of 'indefinite extensibility'. (Burgess, 2004, p. 205)

Further charges of obscurity are leveled by (Oliver, 1998).

Even Ian Rumfitt, who is sympathetic to many Dummettian ideas, expresses reservations. Thus, in an attempt to reconstruct a related Dummettian argument, he seeks to "eschew the rather dark notion of "indefinite extensibility'" (Rumfitt, 2015, p. 264).

\section{Towards an autonomous analysis}

It cannot be denied that Dummett's discussion is somewhat obscure. But I deny that it is hopelessly so. We are presented with some rich and intuitive ideas of great potential fruitfulness - though clearly in need of formal and theoretical development. This ought to whet our philosophical appetite, not make us run away, like scared children, to the perceived safety of set theoretic orthodoxy. Let us be more courageous!

To make things precise, I shall use second-order logic, which extends ordinary firstorder logic by permitting quantification into predicate position. Consider the statement that Socrates thinks, which we formalize as 'Think(Socrates)'. While first-order logic allows us to generalize into the noun position to conclude ' $\exists x \operatorname{THINK}(x)$ ', second-order logic additionally

\footnotetext{
${ }^{6}$ See e.g. (Dummett, 1991), (Dummett, 1993), (Santos, 2013).

${ }^{7}$ For the record, I believe (a), while exaggerated, contains a grain of truth. I reject (c) for reasons that will become clear; see also (Linnebo, 2006).
} 
allows us to generalize into the predicate position to conclude that there is a concept $F$ under which Socrates falls: ' $\exists F F$ (Socrates)'. Throughout, upper-case variables will be secondorder. We shall occasionally also use so-called "lambda notation" and thus write ' $\lambda x . \varphi(x)$ ' for the concept (presumed to be) defined by the open formula $\varphi(x)$.

Any kind of collection can now be represented by means of the corresponding Fregean concept, that is, the concept that holds of $x$ just in case $x$ is a member of the collection. Special kinds of collection can be represented by means of concepts that satisfy suitable special conditions. In this way, we can now provide a more precise analysis of what it is for a concept to be indefinitely extensible. Let us assume, for now, that we have articulated a notion of a concept's being definite -intuitively, that its instances can be "circumscribed". Let us write ' $D(X)$ ' to indicate that the concept $X$ is definite. Relative to this undefined notion of definiteness, it is straightforward to define Dummett's notion of indefinite extensibility.

A concept $F$ is indefinitely extensible just in case there is a function $\delta$ such that:

$$
\forall X(D(X) \wedge \forall x(X x \rightarrow F x) \rightarrow F(\delta(X)) \wedge \neg X(\delta(X)))
$$

We say that $\delta$ is a diagonal function for the concept $F .{ }^{9}$

Of course, it remains to define, or at least to gloss, the notion of definiteness on which our analysis relies.

It is useful to begin by considering some toy models of the notions we are trying to explicate. Working against the background of ordinary ZFC set theory, it is easy to provide some examples.

(i) $X$ is definite iff it is finite and indefinitely extensible iff it is infinite.

(ii) For any cardinal number $\kappa, X$ is definite iff its cardinality is strictly less than $\kappa$ and indefinitely extensible iff its cardinality is greater than or equal to $\kappa$.

(iii) $X$ is definite iff it is set-sized and indefinitely extensible iff it is proper class-sized.

\footnotetext{
${ }^{8}$ As Frege and many of his follows, I shall refer to the value of a second-order variable as a 'concept'. I do this merely for ease of communication and remain neutral on the metaphysics of these "concepts". In particular, I am not committed to their being located in some "third realm" or at the level of Fregean Bedeutung rather than Sinn.

${ }^{9}$ Notice that the diagonal function needs to be understood intensionally, not extensionally as, say, some set-theoretic construct.
} 
Examples (i) and (iii) are the extreme cases, where 'definite' is defined, respectively, as strictly and as liberally as possible. In fact, both extremes have had their defenders: Dummett contends that a concept is indefinitely extensible just in case it is infinite, and Russell conjectured that a concept is indefinitely extensible just in case it is coextensive with the ordinals and thus just in case it is has the size of a proper class. ${ }^{10}$

What is the significance of these toy models? Peter Clark is said to have endorsed (iii) as an analysis and thus to have sought to reduce the notion of indefinite extensibility to a piece of ordinary set theory. ${ }^{11}$ Less obviously, the widely discussed analysis of (Shapiro and Wright, 2006) ends up in the same place, in light of the following fact.

Fact 1 The analysis of (Shapiro and Wright, 2006) is equivalent to (iii) modulo second-order ZFC set theory. ${ }^{12}$

So here too indefinite extensibility is reduced to a notion definable in ordinary set theory.

These reductionist approaches to indefinite extensibility are unacceptable, at least if we want the notion to live up to the great promises made on its behalf. In particular, the notion is supposed to provide an explanation of set theory and of permissible abstraction. Plainly, this work cannot be done if the notion is reduced to a piece of set theory. Dummett, following Cantor and Russell, is therefore to be commended for seeking an analysis of indefinite extensibility which has greater autonomy vis-à-vis ordinary set theory and thus has at least has the possibility of delivering on the ambitious explanatory aims just mentioned. The question is how to give the desired analysis.

Let us begin by recalling the familiar logical distinction between intension and extension. An intension, together with the circumstances, is ordinarily thought to determine an extension. The intension of the word 'philosopher', for example, together with the actual circumstances, determines some objects that are all and only the philosophers, say, $a_{1}, a_{2}, \ldots, a_{m}$. An intension is therefore conceptual, since it picks out some objects on the basis of some

\footnotetext{
${ }^{10}$ See (Russell, 1906, p. 36), as well as (Shapiro and Wright, 2006, Sect. 2) for discussion.

${ }^{11}$ See (Shapiro and Wright, 2006, pp. 274-75), who cite a talk given by Clark in 2000.

${ }^{12}$ This equivalence requires the axiom of Global Choice. Even without this axiom, (iii) is equivalent to a slightly weaker notion of indefinite extensibility, namely:

(IE-weak)

$D(X) \wedge \forall x(X x \rightarrow F x) \rightarrow \exists x(F x \wedge \neg X x)$
} 
characteristic of these objects. It is also modally non-rigid: depending on the circumstances, different objects will possess the relevant characteristic. An extension, by contrast, can be regarded as just the objects that the intension picks out. Thus, in the above example, the extension is "nothing over and above" the plurality consisting of $a_{1}, a_{2}, \ldots, a_{m}$. It follows that the extension, in our present sense, is modally rigid: it has the same members in all the circumstances in which it exists at all. ${ }^{13}$ It is this rigidity that enables us to compare extensions across different circumstances. For example, while the intension philosopher in fact picks out $a_{1}, a_{2}, \ldots, a_{m}$, it might have picked out some other objects. When we make this comparison, we rely on the fact that $a_{1}, a_{2}, \ldots, a_{m}$ comprise the very same objects at the two different circumstances.

The central idea to be pursued in the remainder of this article, which I believe is at least implicit in Dummett's writings, is the following. ${ }^{14}$

\section{Definiteness as having an extension}

A concept $F$ is definite just in case $F$ has an extension. Thus, IE is at heart the phenomenon that a concept can lack an extension even though its intension is perfectly sharp.

Our task is to explicate what it is for a concept to have a sharp intension and to have an extension, and to explain how - surprisingly - a sharp intension may nevertheless fail to determine an extension.

\section{Two ways to develop the analysis}

Before considering Dummett's development of the central idea of definiteness as a matter of having an extension, it will be useful, by way of contrast, to briefly consider another development of the idea.

Suppose we adopt a generative approach to ontology, as we have encountered in Russell and Dummett but also find in the influential iterative conception of sets. According to this conception, sets are generated in stages by successive application of the "set of" operation to some objects available at an earlier stage; for example, when this operation is applied

\footnotetext{
${ }^{13}$ See (Linnebo, 2016) for further discussion.

${ }^{14}$ See (Dummett, 2007), p. 787 and p. 790, where Dummett appears to equate a concept's being definite with its having an extension.
} 
to the objects $a_{1}, \ldots, a_{m}$, we obtain the set $\left\{a_{1}, \ldots, a_{m}\right\}$. An obvious question is how the generative vocabulary should be understood. Presumably, we are not literally bringing sets into existence, as traditional constructivists would have it. (Parsons, 1977) proposes an alternative explication in modal terms. While the existence of the set metaphysically depends on the existence of each of its elements, its own existence is merely potential relative to that of its elements. This idea can be spelled out in the form of a modal set theory, as shown by (Parsons, 1983b) and developed further by (Linnebo, 2013) and (Studd, 2013).

Against this background, it is natural to adopt the following explication of what it is for a concept to have an extension (and thus to be definite and "circumscribable").

\section{Extensions are completable (Parsons, Linnebo, Studd)}

A concept has an extension just in case it is possible for the concept to have some instances such that it is impossible for it to have yet further instances.

For example, the concept of a finite set can be completed (namely at stage $\omega$ ), whereas the concept of an ordinal number cannot, since there are ordinals of arbitrarily high rank.

Dummett proposes an alternative development of the central idea of definiteness as a matter of having an extension. To understand this alternative, it crucial to distinguish two notions of definiteness, which I shall now gradually clarify. First, let us say that a concept $F$ is intensionally definite - symbolized as ' $\mathrm{ID}(F)$ ' - just in case it has a sharp application condition. Intuitively, we may think of this as a matter of our possessing an " $F$-detector", which can freely be moved from one environment to another, and which always yields a clear and definitive verdict as to whether any object that we might come across is or is not an $F$. The concept of being an ordinal, for example, is intensionally definite, whereas the concept of being bald is not. Second, let us say that a concept $F$ is extensionally definite - symbolized as ' $\mathrm{ED}(F)$ ' - just in case it has some fixed extension in all the circumstances in which the concept is available. The paradigm examples are concepts whose instances can be exhaustively listed. For instance, the concept of being one of $a_{1}, \ldots, a_{n}$ is extensionally definite.

Dummett regrettably fails to distinguish clearly between the two notions of definiteness. The distinction is nevertheless implicit in his discussion. He sometimes has in mind what I call "intensional definiteness". Here is one example. 
A concept is definite provided that it has a definite criterion of application - it is determinate what has to hold good of an object for it to fall under the conceptand a definite criterion of identity - it is determinate what is to count as one and the same object. (Dummett, 1991, p. 314)

Dummett goes on to claim that the concepts of set and ordinal are definite in this sense:

We know well enough what is needed for something to be recognised as a set or as an ordinal number, and when an entity given in a certain way is the same set or ordinal number as one given in some another' (p. 315).

But he denies that these concepts correspond to "definite totalities". So the concepts are definite in one sense but not in another. In my terminology, the concepts are intensionally but not extensionally definite. This crucial point is repeated elsewhere in Dummett's writings as well. He writes, for example, that it is possible to have "a clear grasp" of a concept without having attained "a conception of the totality of everything that falls under it" (Dummett, 2007, p. 787).

The most distinctive part of Dummett's view consists of an analysis of what it is for a concept to have an extension. This analysis involves an alleged connection between extensional definiteness and the availability of a "classical interpretation" of quantification: ${ }^{15}$

a definite totality is one quantification over which always yields a statement determinately true or false (Dummett, 1991, p. 316)

So let us consider how Dummett understands this classical interpretation of quantification. The following passage is instructive.

We cannot take quantification over the totality of all objects as a sentence-forming operation which will always generate a sentence with a determinate truth-value; we cannot, in other words, interpret it classically as infinitary conjunction or disjunction. (Dummett, 1981, p. 530)

This suggests that a classical interpretation of quantification is one which seeks to reduce quantification to infinitary conjunctions or disjunctions, or at least to explain the former

\footnotetext{
${ }^{15}$ Cf. also (Dummett, 1981, pp. 529-30) and (Dummett, 1993, pp. 441-42).
} 
in terms of the latter. ${ }^{16}$ We observe that this interpretation supports classical logic for the quantifiers - provided that the instances of the quantified statement are themselves subject to classical logic. For when the instances obey the Law of Excluded Middle (henceforth, 'LEM'), then provably so too do their conjunctions and disjunctions.

Dummett's approach can therefore be summarized as follows.

\section{Extensions as permissible domains for classical quantification (Dummett)}

A concept has an extension (that is, it is ED) just in case quantification over its instances can be interpreted classically.

Before presenting our official analysis of the two forms of definiteness, let us briefly return to the notion of indefinite extensibility. We recall that Dummett's explanation of this notion relies on an undefined notion of definiteness. This undefined notion is clearly extensional definiteness. When this notion is plugged into the Dummettian definition formulated in the previous section, we obtain that $F$ is indefinitely extensible iff:

$$
\forall X(\mathrm{ED}(X) \wedge \forall x(X x \rightarrow F x) \rightarrow F(\delta(X)) \wedge \neg X(\delta(X)))
$$

It follows immediately that an indefinitely extensible concept cannot be ED. ${ }^{17}$ By contrast, many indefinitely extensible concepts are ID; for example, the concepts of an ordinal and of a set.

Thus, the existence of indefinitely extensible concepts would show that the combination of being ID but not ED is realized; that is, that there are concepts which, despite having a sharp intension fail to determine an extension. This would be a surprising discovery, at odds with the dominant view of intensions and extensions described in the previous section.

\footnotetext{
${ }^{16}$ What kind of reduction or explanation does Dummett have in mind? As Ian Rumfitt reminds me, the proposed interpretation is deeply implausible as a piece of philosophy of language. As Frege astutely observes: "If I utter a sentence with the grammatical subject 'all men', I do not wish to say something about some Central African chief wholly unknown to me" (Frege 1895, 454). I shall shortly develop Dummett's suggestion in a more metaphysical direction, which I find more plausible (whether or not this corresponds to Dummett's intention).

${ }^{17}$ If the concept, say $F$, was ED, we could apply the diagonal function $\delta$ to obtain an object, namely $\delta(F)$, which both is and is not $F$.
} 


\section{Formalizing the Dummettian analysis}

We have made substantial progress. We have extracted from Dummett a reasonably clear analysis of indefinite extensibility as involving the failure of a sharp concept to have an extension. And possession of an extension, in turn, has been analyzed in terms of the availability of a classical conception of quantification. But we can do even better. The analysis can be made formally precise, in a very satisfying way. Doing so is useful, for at least two reasons. It will conclusively put to rest any charge of obscurity. And the formal analysis will aid our restruction of Dummett's arguments against classical logic and realism by allowing these arguments to be stated with proper clarity and surprising succinctness.

Our formal analysis takes place against the background of intuitionistic second-order logic. We shall return to the important question of why precisely this sub-classical system should be our background logic.

In the context of intuitionistic logic, it is natural to define intensional definiteness as follows.

Definition 1 A concept $F$ is intensionally definite (in symbols, ' $\left.\operatorname{ID}(F)^{\prime}\right)$ iff $\forall \vec{u}(F \vec{u} \vee \neg F \vec{u})$. Because intuitionistic logic proves $\varphi \rightarrow \neg \neg \varphi$, Definition 1 ensures:

$$
\mathrm{ID}(X) \rightarrow \operatorname{ID}(\neg X)
$$

(However, the converse fails, as $\neg \varphi \vee \neg \neg \varphi$ does not entail $\varphi \vee \neg \varphi$ in intuitionistic logic.) Likewise, we can prove that intensional definiteness is preserved under conjunction and disjunction.

We now set out some principles of definiteness that are at least implicit in Dummett's discussion.

Principle 1. If $X$ is ED, then $X$ is ID.

That is, the ED concepts form a proper subclass of the ID ones. Notice that Principle 1 entails that if $X$ is $\mathrm{ED}$, then $\neg X$ is ID.

Next, we have: 
Principle 2. Any empty concept is ED. Moreover, adjunction of an $n$-tuple to an $n$-adic ED concept preserves ED-ness: $\operatorname{ED}(X) \rightarrow \operatorname{ED}(\lambda \vec{x} .(X \vec{x} \vee \vec{x}=\vec{a}))$.

There are two reasons to ascribe this two-pronged principle to Dummett. First, he believes that finite collections are "definite totalities" and thus correspond to ED concepts. Principle 2 provides a natural and systematic expression of this belief. Second, Dummett assumes that every indefinitely extensible concept $F$ gives rise to a sequence of extensions produced by iterated application of the diagonal function $\delta$. Consider an ED collection $X$ of $F$ s. By applying $\delta$ to $X$, we obtain $\delta(X)$, which is an $F$ that is not in $X$. Thus, by adjoining this new $F$ to $X$, we obtain a strictly larger collection of $F$ s. To apply $\delta$ again and thus continue the sequence, the operation of adjunction needs to preserve extensional definiteness.

Our next principle is reminiscent of the set-theoretic axiom of Separation. ${ }^{18}$

Principle 3. Assume $X$ is ED and $Y$ is ID. Then the intersection of the two concepts, namely $\lambda \vec{x} .(X \vec{x} \wedge Y \vec{x})$, is ED.

This principle is suggested by the following passage:

it must be allowed that every concept defined over a definite totality determines a definite sub-totality ${ }^{19}$

The principle is also implicit in Dummett's discussion of how the ID concept of a star gives rise to a "definite totality" of stars when applied to a "definite totality" of celestial bodies. ${ }^{20}$

The final - and most interesting — principle concerns the connection Dummett sees between extensional definiteness and the availability of a classical interpretation of quantification. $^{21}$ Suppose quantification restricted to some concept $F$ can be interpreted classically. Then presumably such quantification also "behaves classically". This suggests that we can characterize - and perhaps even define - what it is for F to be ED in terms of the "classical behavior" of quantification restricted to $F$.

Let us begin our exploration of this suggestion by asking what it means for quantification restricted to $F$ to "behave classically". A natural answer is that statements based on such

\footnotetext{
${ }^{18}$ Separation is an axiom scheme of ordinary ZFC set theory which ensures that, for any set $a$ and formula $\varphi(x)$, there exists a set of all the elements of $a$ which satisfy $\varphi(x)$, i.e. $\{x \in a: \varphi(x)\}$.

${ }^{19}$ See (Dummett, 1991, 317); cf. also (Dummett, 1981, p. 530).

${ }^{20}$ See (Dummett, 1991, p. 314) and (Dummett, 1993, p. 438).

${ }^{21}$ See the previous section for some relevant quotes and further references.
} 
quantification are subject to LEM: ${ }^{22}$

$$
(\forall x: F x) \varphi(x, \vec{y}) \vee \neg(\forall x: F x) \varphi(x, \vec{y})
$$

As a general requirement, however, (2) would be too strong: non-classical behavior might sneak in via $\varphi$. Thus, we should only require (2) on the assumption that:

$$
\forall x \forall \vec{y}(\varphi(x, \vec{y}) \vee \neg \varphi(x, \vec{y}))
$$

We now have an answer to our question. For the quantification restricted to $F$ "to behave classically" is for it to preserve intensional definiteness; that is, if $\lambda x \lambda \vec{y} \cdot \varphi(x, \vec{y})$ is ID, then so is $\lambda \vec{y} .(\forall x: F x) \varphi(x, \vec{y})$.

Next, let us inquire under what conditions quantification restricted to $F$ does behave classically, in the sense just articulated. We begin with a partial answer. Let us say that a concept $F$ is finitely traversable when there are constants $c_{i}$ such that:

$$
\forall x\left(F x \leftrightarrow x=c_{0} \vee \ldots \vee x=c_{n}\right)
$$

Let us also adopt the following axiom:

Axiom 1. The identity predicate is ID.

Given this axiom, it is easy to show that (3) and (4) entail (2). That is, quantification restricted to any finitely traversable concept $F$ behaves classically.

This partial answer can now be generalized. Let us say that a concept $F$ is traversable just in case there are, or we can introduce, constants $c_{\gamma}$ such that:

$$
\forall x\left(F x \leftrightarrow \bigvee_{\gamma \in \Gamma} x=c_{\gamma}\right)
$$

Assume $F$ is traversable. Then quantification restricted to $F$ reduces to a (perhaps infinitary) disjunction or conjunction, namely, $(\forall x: F x) \varphi(x, \vec{y}))$ reduces to $\bigwedge_{\gamma \in \Gamma} \varphi\left(c_{\gamma}, \vec{y}\right)$. And this, in turn, ensures that quantification restricted to $F$ behaves classically. This insight is expressed

${ }^{22}$ As usual, ' $(\forall x: F x) \varphi$ ' is shorthand for ' $\forall x(F x \rightarrow \varphi)$.' 
compactly by the following principle:

Principle 4. $\quad \operatorname{ED}(X) \wedge \operatorname{ID}(Y) \rightarrow \operatorname{ID}(\lambda \vec{v} .(\forall \vec{u}: X \vec{u}) Y \vec{u} \vec{v}))$.

In fact, Dummett's discussion suggests something even stronger. As we have seen, he characterizes "a definite totality" - which corresponds to our notion of an extensionally definite concept - as "one quantification over which always yields a statement determinately true or false" (Dummett, 1991, p. 316). This sounds like a connection that is tight enough to be regarded as definitional. Accordingly, let us attempt the following definition of extensional definiteness.

Definition 2 Let us say that a concept $X$ is extensionally definite iff:

(i) $X$ is intensionally definite

(ii) quantification restricted to $X$ preserves intensional definiteness:

$$
\forall Y(\operatorname{ID}(Y) \rightarrow \operatorname{ID}((\forall \vec{u}: X \vec{u}) Y \vec{u}))
$$

where the adicity of $Y$ is the same as that of $X .^{23}$

This definition turns out to be very fruitful. For one thing, it enables us to prove the following pleasing result.

Theorem 1 Assume intuitionistic logic and Axiom (I). Then our definitions of ID and ED entail all of the discussed principles of definiteness.

For another, the definition entails further useful and plausible principles of definiteness, for example, principles of Union and Replacement for extensional definiteness. ${ }^{24}$

Let us take stock. We began with the complaint that Dummett's notion of indefinite extensibility is hopelessly obscure. We have offered a mixed assessment. The complaint is half wrong - because Dummett provides a perfectly good definition of indefinite extensibility

\footnotetext{
${ }^{23}$ In fact, (ii) is equivalent to the prima facie stronger requirement that for any $Y$ of adicity greater than or equal to that of $X$, if $\operatorname{ID}(Y)$, then $\operatorname{ID}(\lambda \vec{v}(\forall \vec{u}: X \vec{u}) Y \vec{u} \vec{v})$. The proof of this equivalence is not hard, using the fact that $\operatorname{ID}(\lambda \vec{u} Z \vec{u}) \leftrightarrow \forall \vec{u} \operatorname{ID}(Z \vec{u})$ for any concept $Z$ of any adicity.

${ }^{24}$ See Appendix A for proofs of the theorem and these further claims. In fact, the Dummett-inspired approach appears to entail more principles of extensional definiteness than the alternative approach based on the modal notion of completability described in Section 5 .
} 
in terms of a notion of definiteness - but also half right - because this notion of definiteness isn't properly spelled out. Even so, we have managed to extract precise definitions of ID and ED from Dummett's discussion. These definitions can serve in an account of indefinite extensibility, where they allow us to prove all of the principles of definiteness that Dummett (explicitly or implicitly) assumes.

\section{Extensional definiteness and abstraction}

Following Cantor and Russell, Dummett assumes that ED concepts define sets, have order types, and figure in other forms of abstraction as well. This assumption is implicit in his use of the diagonal function $\delta$ to proceed from some "definite totality" $X$ of $F$ s to another $F$ that lies beyond of this "totality". ${ }^{25}$

It suffices for present purposes to consider the case of sets. Let ' $\operatorname{SET}(F, x)$ ' stand for ' $F$ defines the set $x$ '. We can then explicate Dummett's assumption in the form of a two-part axiom, which states that ED concepts define sets and that sets obey the law of extensionality:

$$
\begin{gathered}
\operatorname{ED}(F) \rightarrow \exists x \operatorname{SET}(F, x) \\
\operatorname{Set}(F, x) \wedge \operatorname{SET}(G, y) \rightarrow(x=y \leftrightarrow \forall u(F u \leftrightarrow G u))
\end{gathered}
$$

Further, we let $x \in y$ iff $y$ is the extension of some ED concept under which $x$ falls. That is, we define ' $x \in y$ ' as ' $\exists F(\operatorname{ED}(F) \wedge \operatorname{SET}(F, y) \wedge F x)$ '.

One of Dummett's central arguments is now easily set out. We begin with the following observation.

Fact 2 If Axiom (II) is accepted, then LEM is invalid.

Proof. Suppose all instances of LEM are valid. Then all concepts are ID and thus also ED. In the presence of (II), this yields Russell's paradox. $\dashv$

Since Axiom (II) seems plausible, we are thus meant to conclude that classical logic must be rejected. We shall return to the question of how dialectically effective the argument is. (It is

\footnotetext{
${ }^{25}$ It is unclear (to me at least) whether Dummett believes that certain forms of abstraction are permissible on ID concepts as well, irrespective of whether the concept is ED.
} 
not obvious why we should point the finger at LEM rather than some other part of classical logic.)

Let us adopt another axiom:

$$
\operatorname{ID}(\lambda x . x \notin x)
$$

This axiom is implicit in Dummett characterization of the concept of a set that is not an element of itself as "a beautiful example of an indefinitely extensible concept" (Dummett, 1993, p. 441).

We are now finally able to prove the existence of indefinitely extensible concepts.

Fact 3 If (II) and (III), then the concept of a set is indefinitely extensible.

Proof. Assume $X$ is an ED concept contained in the concept of a set. By (III), the "Russell concept" relative to $X$, namely $\lambda x .(x \notin x \wedge X x)$, is also ED. (Here we use Principle 3.) By (II), the latter concept defines a set. We define a function $\delta$ from concepts to objects by letting $\delta(X)$ be this set, if $X$ satisfies the assumption with which we began, or the empty set, if not. Suppose, for contradiction, that $X$ satisfies the mentioned assumption and $\delta(X)$ falls under $X$. We prove that $\delta(X) \in \delta(X)$ iff $\delta(X)$ falls under $\lambda x .(x \notin x \wedge X x)$, iff $\delta(X) \notin \delta(X)$, which is a contradiction. Thus, the set $\delta(X)$ isn't in $X$. This means that the concept of a set is IE. $\dashv$

\section{Extensional definiteness and realism}

Suppose realism is defined in terms of the validity of LEM, as Dummett is inclined to do. Then Fact 2 refutes realism as well as classical logic.

However, Dummett's discussion containts another argument against realism as well, which is more interesting. According to the realist, Dummett writes, a

concept is definite provided that it has a definite criterion of application - it is determinate what has to hold good of an object for it to fall under the conceptand a definite criterion of identity - it is determinate what is to count as one and the same such object. [...] We do not need to be able to say just what objects 
there are which fall under the given concept: provided the concept is definite, reality will of itself determine the truth or falsity of such [quantified] statements. ${ }^{26}$

Suppose, for example, that we give a definition of 'star' which is sharp enough to yield an ID concept. And suppose realism is appropriate for the domain of astronomy. When the mentioned concept is brought to bear on this domain, astronomical reality answers with a well-defined range of objects, namely the totality of stars.

The observation generalizes. On the assumption of realism, any ID concept suffices to determine a "definite totality" of objects. When such a concept is brought to bear, reality answers with a well-defined range of instances. And since it is well-defined, this range can serve as a domain for classically interpreted quantification. These considerations are summed up by the following axiom:

$$
\text { REALISM } \rightarrow \forall F(\mathrm{ID}(F) \rightarrow \mathrm{ED}(F))
$$

Dummett's attempted refutation of realism can now be developed as follows.

Fact 4 If (II), (III), and (IV), then $\neg$ REALiSM.

Proof. By (III), the concept $R$, defined by $x \notin x$, is ID. Assume Realism. Then (IV) entails that $R$ is also ED. So by (II), $R$ defines a set, which yields Russell's paradox. $\dashv$

\section{$9 \quad$ Assessing the Dummettian arguments}

The two Dummettian arguments just presented raise many questions. The following three are particularly pressing.

(1) Do our definitions of intensional and extensional definiteness capture their intended targets?

(2) Why accept the four axioms that we have formulated?

(3) Why let the background logic be intuitionistic? Why not classical logic or perhaps some logic intermediate between intuitionistic and classical?

\footnotetext{
${ }^{26}$ See (Dummett, 1991, p. 314); cf. also (Dummett, 1993, p. 439).
} 
There is surprisingly little explicit discussion of these questions in Dummett. So at best, he provides argument templates, not complete arguments.

When discussing these questions, I shall obviously speak for myself, not (even partially) on behalf of Dummett.

\subsection{The definitions of intensional and extensional definiteness}

For now, let us hold fixed the assumption that the correct background logic is intuitionistic. Then, I contend, a strong case can be made for the appropriateness of our definitions of intensional and extensional definiteness.

It is natural to understand the intuitionistic logic in terms of Kripke models whose nodes represent the stage of our generative process. ${ }^{27}$ We define, in the usual way, what it is for a formula $\varphi$ to "be forced" by a node $s$ relative to a variable assignment $\sigma$; we symbolized this as $s \Vdash_{\sigma} \varphi$. And as usual, whatever is forced by one stage remains forced by any extension of this stage:

$$
\text { if } s \leq s^{\prime} \text { and } s \Vdash_{\sigma} \varphi \text {, then } s^{\prime} \Vdash_{\sigma} \varphi
$$

It is convenient to write $s \Vdash \varphi\left(a_{0}, \ldots, a_{n}\right)$ as shorthand for $s \Vdash_{\sigma} \varphi$, where $\sigma\left(x_{i}\right)=a_{i}$ for each variable $x_{i}$ that occurs free in $\varphi$.

First, consider the definition of intensional definiteness. It is straightforward to verify that $s \Vdash \operatorname{ID}(F))$ iff:

$$
\left(\forall s^{\prime} \geq s\right)\left(\forall \vec{a} \in D\left(s^{\prime}\right)\right)\left(\forall s^{\prime \prime} \geq s^{\prime}\right)\left(s^{\prime} \Vdash F \vec{a} \text { iff } s^{\prime \prime} \Vdash F \vec{a}\right)
$$

The displayed formula provides a good analysis of what it means to "have an $F$-ness detector". Consider any environment $s^{\prime}$ that is richer than $s$ and any string of objects $\vec{a}$ that can be found at $s^{\prime}$. Then we obtain a clear verdict as to whether $F \vec{a}$, namely $s^{\prime} \Vdash F \vec{a}$. And this verdict is definitive because it cannot be overturned in any yet richer environment $s^{\prime \prime}$.

Next, consider the definition of extensional definiteness. Let us say that a concept $F$ is complete at $s$ just in case no additional $F$ s become available at any later stage $s^{\prime} \geq s$. Given some plausible assumptions, we can show that $F$ is ED at $s$ just in case $F$ is both ID and

\footnotetext{
${ }^{27}$ Readers unfamiliar with Kripke models for intuitionistic logic should either change that (e.g. by consulting (Moschovakis, 2015)) or else take my contention on faith (and may thus skip this subsection).
} 
complete at $s .^{28}$ This means that a concept is ED at $s$ just in case its instances are complete, and thus circumscribed, by $s$.

\subsection{Axioms (I) and (III)}

Let us continue to assume that the background logic is intuitionistic.

Axioms (I) and (III) state that the concepts of identity and non-self-membership are ID. Both axioms are plausible - at least on the interpretation of the generative process that I favor. To "generate" an object is simply a matter of providing clear and definite answers to every question about the object which can be formulated using the identity predicate or any other atomic predicate. The case of sets provides a good illustration. Consider some objects $a a$. When we "generate" their set, we lay down that any question of the form $\{a a\}=\{b b\}$ (or $b \in\{a a\}$ ) is to be answered in accordance with whether or not $a a$ and $b b$ are the very same objects (or whether or not $b$ is one of $a a$ ).

In fact, this interpretation motivates the following strengthening of Axiom (III):

$$
\operatorname{ID}(\in)
$$

Thus, we obtain a Dummett-inspired set theory based on intuitionistic second-order logic and Axioms (I), (II), and $\left(\mathrm{III}^{+}\right) \cdot{ }^{29}$

\subsection{Axiom (IV)}

Axiom (IV), we recall, states:

$$
\mathrm{REALISM} \rightarrow \forall F(\mathrm{ID}(F) \rightarrow \mathrm{ED}(F))
$$

The plausibility of this axiom obviously depends on how "realism" is understood. There are many options.

\footnotetext{
${ }^{28}$ See Appendix B for details.

${ }^{29}$ This set theory is related to Solomon Feferman's "semi-constructive set theory"; see (Rathjen, 2016, Sections 1 and 2) for a useful explanation and further references, including to Feferman's (published and unpublished) works. In particular, in both theories, global quantification is intuitionistic, while quantification restricted to any set is classical. Notice, moreover, that the principles of definiteness proved in Appendix B enable us to prove versions of the familiar set-theoretic axioms of Separation, Union, and Replacement.
} 
If realism is solely a matter of the validity of LEM, as Dummett is inclined to think, then the question of the plausibility of Axiom (IV) is moot. For then Axiom (II) alone suffices to refute realism, eliminating the need for (IV).

Another relevant form of realism is "metaphysical realism", as characterized by Putnam, namely the view that the world is partitioned into a range of objects in a unique and objective way. ${ }^{30}$ This form of realism supports Axiom (IV). When an ID concept is applied to this all-encompassing range of objects, we obtain a well-defined range of instances, on which Dummett's classical interpretation of quantification is therefore defined. Thus, Dummett's argument poses a threat to this form of realism. (Whether the argument succeeds will of course depend on the status of its remaining assumptions.)

While this threat to metaphysical realism is real, it is hardly surprising. For there is very little conceptual distance between Putnam's metaphysical realism and the rejection of indefinite extensibility. Indeed, on this version of realism, Axiom (IV) is little more than a truism. I don't know whether there are forms of realism which conflict with the existence of indefinite extensibility in a less direct (and therefore more surprising) way.

More importantly, there are versions of realism on which Axiom (IV) is false. Indeed, in Section 9.5 I canvass a robust form of realism which is perfectly compatible with the existence of indefinitely extensible concepts.

\subsection{Axiom (II)}

Consider now Axiom (II), which states that every ED concept corresponds to a set.

By Fact 2, this axiom is classically inconsistent. An obvious question is therefore whether the corresponding intuitionistic theory is consistent. Fortunately, we can prove that it is. ${ }^{31}$

Theorem 2 Assume classical ZFC. Then the set theory based on intuitionistic logic and Axioms (I), (II), and $\left(\mathrm{III}^{+}\right)$is consistent.

Next, we ask what positive reason there is to accept Axiom (II). Let us continue to assume

\footnotetext{
${ }^{30}$ Metaphysical realism is a recurring bête noire in Putnam's writings; see e.g. (Putnam, 1987).

${ }^{31}$ Since the proof is more involved than those of the other results of this article, a proper presentation is reserved for other work. (The proof strategy is to (i) start with a Kripke model of modal set theory, as described in (Linnebo, 2013); (ii) convert this model to one that is "capricious" in the sense of Appendix B; and (iii) apply so-called Beth-Kripke semantics for intuitionistic logic.)
} 
that the background logic is intuitionistic. Then, as argued in Section 9.1, any ED concept is complete. This ensures that it is possible to define the set of all instances of the concept. Intuitively, when a concept is complete, there is "room above" all of its instances in which to locate the set of precisely these objects. ${ }^{32}$

\subsection{The choice of background logic}

This brings us to the hardest, but also the most interesting, question. Why let our background logic be intuitionistic?

Of course, given Axiom (II), Fact 2 shows that the logic cannot be classical. But our defense of (II) was conditional on the assumption that the logic is intuitionistic! All we have established is therefore that (II) and intuitionistic logic form a natural "package deal": given intuitionistic logic, (II) is plausible; and given (II), the logic cannot be classical but can be intuitionistic. Let us call this the Dummett package.

There is also an alternative package deal, namely the Parsons-Linnebo-Studd package described in Section 5. This alternative package combines classical logic with (a modal formulation of) the assumption that all completable concepts define sets. Because the logic is classical, this package is far more faithful to orthodox set theory. Moreover, no compelling reason has yet been offered for abandoning this package in favor of Dummett's more heterodox alternative.

I wish to end by canvassing a possible reason to adopt the heterodox alternative. Consider a true generalization $\forall x \varphi(x)$. What explains, or grounds, this truth? Suppose we insist on a substantive answer to this question. A natural option is to give an instance-based explanation. The generalization is true because each of its instances is true and, perhaps, because these are all the instances. This conception of what grounds a universal generalization can be seen as a metaphysical correlate of Dummett's classical interpretation of quantification. The instance-based explanation is intuitive and underwrites classical logic whenever it is available. The problem is that such explanations aren't always available. When the domain isn't ED, there is no well-defined range of instances to consider. In such cases, we need an alternative, non-instance-based explanation of true generalizations.

\footnotetext{
${ }^{32}$ See (Linnebo, 2013) for details.
} 
What might explain a universal generalization, if not its instances? The only answer known to Dummett involved the form of anti-realism he found so attractive, namely, that the truth of the generalization is to be understood in terms of the existence - or perhaps even our possession - of a proof of it. As is well known, this anti-realist conception supports intuitionistic logic. ${ }^{33}$ But needless to say, the anti-realism on which this answer relies is extremely controversial.

Happily, the appeal to anti-realism is not obligatory: there are other ways to obtain the desired non-instance-based explanations. An interesting alternative is suggested by Hermann Weyl. What might explain the truth of a universal generalization $\forall n \varphi(n)$ over the natural numbers? (Weyl, 1921, p. 54) observes that the explanation need not proceed via the infinitely many instances but might instead be based on the fact that "it lies in the essence of" the concept of a natural number that each natural number should satisfy $\varphi$. Importantly, this suggestion is fully compatible with realism: there may be robust truths about concepts and their essential connections, which should not be understood in terms of proof. The suggestion is also compatible with appeals to instance-based explanations wherever these are available. I develop a version of Weyl's suggestion in (Linnebo, 2018) and show that this validates semi-intuitionistic logic, that is, intuitionistic logic where quantification restricted to any set behaves classically. Thus, this is only a very mild departure from classical logic.

\section{Conclusion}

To sum up, I have defended four main claims.

First, despite its apparent obscurity, the notion of indefinite extensibility can be articulated in ways that are philosophically illuminating and mathematically interesting.

Second, despite its apparent obscurity, Dummett's alternative argument against realism and for intuitionistic logic can be articulated in a way that is philosophically illuminating and mathematically interesting.

Third, while Dummett's alternative argument threatens certain forms of realism - in particular, what Putnam calls "metaphysical realism" - there are other robust forms of realism

\footnotetext{
${ }^{33}$ This can be seen, for example, by considering the Brouwer-Heyting-Kolmogorov interpretation of the logical operators.
} 
to which the argument poses no threat.

Finally, Dummett's intuitionistic explication of indefinite extensibility has not yet been shown to be preferable to an alternative but thoroughly classical explication. But it might be possible to extend Dummett's alternative argument in a way that threatens LEM and instead validates a semi-intuitionistic logic. If so, friends of classical logic should not be too alarmed, because their favored logic will remain valid whenever the domain is extensionally definite. ${ }^{34}$

\footnotetext{
${ }^{34}$ Thanks to Kit Fine, Bob Hale, Jon Litland, David McCarty, Michael Rathjen, Ian Rumfitt, and audiences in Bucharest, Oslo, Oxford, and Paris for comments and discussion.
} 


\section{Appendices}

\section{A Proofs of some principles of definiteness}

Proof sketch for Theorem 1. Principles 1 and 4 are immediate from the definition of ED. Principle 2 is straightforward. (This is the only place where Axiom (I) is needed.) To prove Principle 3, observe that $X \wedge Y \rightarrow Z$ is intuitionistically equivalent to $X \rightarrow(Y \rightarrow Z)$ and that $Y \rightarrow Z$ is ID provided that $Y$ and $Z$ are. $\dashv$.

In fact, Theorem 1 can be strengthened substantially.

Theorem 3 Assume intuitionistic logic and Axiom (I). Then our definitions of ID and ED enable us to prove the following additional principles of definiteness.

D1. The union of any two ED concepts is itself ED:

$$
\mathrm{ED}(X) \wedge \mathrm{ED}(Y) \rightarrow \mathrm{ED}(\lambda x .(X x \vee Y x))
$$

D2. Extensional definiteness is factorizable, in the sense that a relation is ED iff both its domain and each of its sections is ED:

$$
\operatorname{ED}(R) \leftrightarrow \operatorname{ED}(\lambda \vec{u} \exists \vec{v} R \vec{u} \vec{v}) \wedge \forall \vec{u} \operatorname{ED}(\lambda \vec{v} R \vec{u} \vec{v})
$$

D3. Assume $X$ is ED, $R$ is ID, and that $\lambda y R x y$ is ED for each $x$ such that $X x$. Then the image of $X$ under $R$ is ED as well:

$$
\operatorname{ED}(\lambda y \exists x(X x \wedge R x y))
$$

Proof sketch. D1 follows by observing that $\forall x(X x \vee Y x \rightarrow Z x)$ is intuitionistically equivalent to $\forall x(X x \rightarrow Z x) \wedge \forall x(Y x \rightarrow Z x)$.

For the right-to-left direction of D2, we begin by observing that the following two formulas 
are intuitionistically equivalent:

$$
\begin{gathered}
\forall \vec{u} \forall \vec{v}(R \vec{u} \vec{v} \rightarrow \theta) \\
\forall \vec{u}(\exists \vec{v} R \vec{u} \vec{v} \rightarrow \forall \vec{v}(R \vec{u} \vec{v} \rightarrow \theta))
\end{gathered}
$$

We now reason as follows. Assume $G$ is ID. Since $\lambda v R u v$ is ED, we have:

$$
\forall v(R u v \rightarrow G) \vee \neg \forall v(R u v \rightarrow G)
$$

In other words, $\lambda u .(\forall v(R u v \rightarrow G))$ is ID. Next, since $\lambda u \exists v R u v$ is ED, we have:

$$
\forall u(\exists v R u v \rightarrow \forall v(R u v \rightarrow G)) \vee \neg \forall u(\exists v R u v \rightarrow \forall v(R u v \rightarrow G))
$$

Finally, by the equivalence of (6) and (7), we get our desired conclusion that quantification restricted to $R$ preserves ID-ness:

$$
\forall u \forall v(R u v \rightarrow G) \vee \neg \forall u \forall v(R u v \rightarrow G)
$$

For the left-to-right direction of $\mathrm{D} 2$, assume $\operatorname{ED}(R)$. Let $S$ be the relation defined by $x=a \wedge y=y$; this is ID. So by Principle 3, the intersection of $R$ and $S$ is ED. But this intersection is $\lambda \vec{v} R a \vec{v}$. Since $a$ is arbitrary, we obtain the right-hand conjunct. It remains to prove that $\operatorname{ED}(\lambda \vec{u} \exists \vec{v} R \vec{u} \vec{v})$. Consider an ID concept $G$. We need to show:

$$
\forall \vec{u}(\exists \vec{v} R \vec{u} \vec{v} \rightarrow G \vec{u}) \vee \neg \forall \vec{u}(\exists \vec{v} R \vec{u} \vec{v} \rightarrow G \vec{u})
$$

But this formula follows from our assumption that $\operatorname{ED}(R)$ and the intuitionist equivalence of $\forall \vec{u}(\exists \vec{v} R \vec{u} \vec{v} \rightarrow G \vec{u})$ and $\forall \vec{u} \forall \vec{v}(R \vec{u} \vec{v} \rightarrow G \vec{u})$.

Finally, to prove D3, let $S$ be defined as $\lambda x \lambda y(X x \wedge R x y)$. It is not hard to show that the assumptions of D3 entail $\operatorname{ED}(S)$. Since our target concept $\lambda y \exists x(X x \wedge R x y)$ is a projection of $S$, it follows from D2 that this concept is ED, as desired. $\dashv$ 


\section{B Extensional definiteness vs. completeness}

Let the extension of $F$ at $w$, written $\llbracket F \rrbracket_{w}$, be defined as $\{a \in D(w): w \Vdash F a\}$. Recall that a concept $F$ is said to be "complete at $w$ " iff any instance of $F$ that can be found at any extended world $w^{\prime} \geq w$ is already available at $w$; that is, iff $\llbracket F \rrbracket_{w^{\prime}}=\llbracket F \rrbracket_{w}$ for any $w^{\prime} \geq w$. Let us say that a concept $\tilde{F}$ is a capricious twin of $F$ relative to $w$ iff the following conditions are satisfied:

(i) $\tilde{F}$ is ID at $w$

(ii) there is a $w^{\prime} \geq w$ such that, for any $w^{\prime \prime} \geq w^{\prime}$, we have $\llbracket \tilde{F} \rrbracket_{w^{\prime \prime}}=\llbracket F \rrbracket_{w^{\prime \prime}}$

(iii) there is a $w^{\prime} \geq w$ such that, for any $w^{\prime \prime} \geq w^{\prime}$, we have $\llbracket \tilde{F} \rrbracket_{w^{\prime \prime}}=\llbracket F \rrbracket_{w}$

Intuitively, an ID concept $\tilde{F}$ is a capricious twin of $F$ relative to $w$ iff there is one future in which $\tilde{F}$ behaves precisely like $F$ and another future in which $\tilde{F}$ fails to grow vis-à-vis the extension of $F$ at $w$. Let us say that a Kripke model is capricious iff, for every $w$ and every $F$ that is ID but incomplete at $w$, there is at $w$ a capricious twin $\tilde{F}$ of $F$ relative to $w$.

Theorem 4 Suppose a Kripke model for intuitionistic second-order logic is capricious. Then, for any world $w$ and any $F$ that exists at $w$, we have: $F$ is ED at $w$ iff $F$ is complete at $w$.

Proof sketch. The right-to-left direction is routine. We now prove the contrapositive of the left-to-right direction. Clearly, if $F$ fails to be ID at $w$, it also fails to be ED at $w$. So it suffices to prove that, if a concept $F$ is ID but incomplete at $w$, then $w \forall \operatorname{ED}(F)$. Assume the antecedent. Let $\tilde{F}$ be a capricious twin of $F$ relative to $w$. Our definition ensures that $\tilde{F}$ too is ID at $w$. I claim that:

$$
w \Downarrow \forall \forall x(F x \rightarrow \tilde{F} x) \vee \neg \forall x(F x \rightarrow \tilde{F} x)
$$

To see that $w$ doesn't force the first disjunct, consider the future behavior of $\tilde{F}$ described in (iii) above. To see that $w$ doesn't force the second disjunct, consider the future behavior of $\tilde{F}$ described in (ii) above. $\dashv$ 


\section{References}

Benacerraf, P. and Putnam, H., editors (1983). Philosophy of Mathematics: Selected Readings, Cambridge. Cambridge University Press. Second edition.

Boolos, G. (1998). Logic, Logic, and Logic. Harvard University Press, Cambridge, MA.

Burgess, J. P. (2004). E Pluribus Unum: Plural Logic and Set Theory. Philosophia Mathematica, 12(3):193221.

Cantor, G. (1883). Grundlagen einer allgemeinen Mannigfaltigkeitslehre. B.G. Teubner, Leipzig.

Dummett, M. (1963). The philosophical significance of Gödel's theorem. In Truth and Other Enigmas (1978), pages 186-214. Duckworth.

Dummett, M. (1978). The philosophical basis of intuitionistic logic. In Truth and Other Enigmas, pages 215-247. Harvard University Press.

Dummett, M. (1981). Frege: Philosophy of Language. Harvard University Press, Cambridge, MA, second edition.

Dummett, M. (1991). Frege: Philosophy of Mathematics. Harvard University Press, Cambridge, MA.

Dummett, M. (1993). What is mathematics about? In The Seas of Language, pages 429-445. Oxford University Press.

Dummett, M. (2007). Reply to Peter M. Sullivan. In Auxier, R. E. and Hahn, L. E., editors, The Philosophy of Michael Dummett, The Library of Living Philosophers, pages 786-799. Open Court, Chicago, IL.

Ewald, W. (1996). From Kant to Hilbert: A Source Book in the Foundations of Mathematics, volume 2. Oxford University Press, Oxford.

Fine, K. (2006). Relatively unrestricted quantification. In Rayo, A. and Uzquiano, G., editors, Absolute Generality, pages 20-44. Oxford University Press, Oxford.

Glanzberg, M. (2004). Quantification and Realism. Philosophy and Phenomenological Report, 69:541-72.

Heck, R. G. (1993). Critical notice of Michael Dummett, Frege: Philosophy of Mathematics. Philosophical Quarterly, 43(171):223-233.

Kripke, S. A. (1982). Wittgenstein on Rules and Private Language. Harvard University Press.

Linnebo, Ø. (2006). Sets, properties, and unrestricted quantification. In Rayo, A. and Uzquiano, G., editors, Absolute Generality, pages 149-178. Oxford University Press, Oxford.

Linnebo, Ø. (2013). The potential hierarchy of sets. Review of Symbolic Logic, 6(2):205-228.

Linnebo, Ø. (2016). Plurals and modals. Canadian Journal of Philosophy, 46:654-76.

Linnebo, Ø. (2018). Generality explained. Unpublished MS.

Moschovakis, J. (2015). Intuitionistic logic. In Zalta, E. N., editor, The Stanford Encyclopedia of Philosophy. Spring 2015 edition. 
Oliver, A. (1998). Hazy totalities and indefinitely extensible concepts. Grazer Philosophische Studien, 55:25-50.

Parsons, C. (1974). Sets and classes. Noûs, 8:1-12.

Parsons, C. (1977). What is the iterative conception of set? In Butts, R. and Hintikka, J., editors, Logic, Foundations of Mathematics, and Computability Theory, pages 335-367. Reidel, Dordrecht. Reprinted in (Benacerraf and Putnam, 1983) and (Parsons, 1983a).

Parsons, C. (1983a). Mathematics in Philosophy. Cornell University Press, Ithaca, NY.

Parsons, C. (1983b). Sets and modality. In Mathematics in Philosophy, pages 298-341. Cornell University Press, Cornell, NY.

Priest, G. (1994). The structure of the paradoxes of self-reference. Mind, 103:25-34.

Putnam, H. (1987). The Many Faces of Realism. Open Court.

Rathjen, M. (2016). Indefiniteness in semi-intuitionistic set theories: On a conjecture of Feferman. Journal of Symbolic Logic, 81(2):742-754.

Rumfitt, I. (2015). The Boundary Stones of Thought: An Essay in the Philosophy of Logic. Oxford University Press.

Russell, B. (1906). On some difficulties in the theory of transfinite numbers and order types. Proceedings of the London Mathematical Society, 4:29-53.

Russell, B. (1908). Mathematical logic as based on a theory of types. American Journal of Mathematics, $30: 222-262$.

Santos, G. (2013). Numbers and everything. Philosophia Mathematica, 21(3):297-308.

Shapiro, S. (2003). Prolegomenon to Any Future Neo-Logicist Set Theory: Extensionality and Indefinite Extensibility. British Journal for the Philosophy of Science, 54(1):59-91.

Shapiro, S. and Wright, C. (2006). All things indefinitely extensible. In Rayo, A. and Uzquiano, G., editors, Absolute Generality, pages 255-304. Oxford University Press, Oxford.

Studd, J. (2013). The iterative conception of set: A (bi-)modal axiomatisation. Journal of Philosophical Logic, 42(5):697-725.

Studd, J. P. (201?). Everything, More or Less: A Defence of Generality Relativism. Oxford University Press, Oxford.

Weyl, H. (1921). Über die neue Grundlagenkrise der Mathematik. Mathematische Zeitschrift, 10(1-2):39-79. 